\title{
Effect of Nutritional Restriction on the Hair Follicles Development and Skin Transcriptome of Chinese Merino Sheep
}

\author{
Xuefeng Lv ${ }^{1,2,+}$, Lei Chen ${ }^{3,4, \dagger}$, Sangang He ${ }^{3,4}$ (D) Chenxi Liu ${ }^{3,4}$, Bin Han ${ }^{3,4}$, Zhilong Liu ${ }^{3,4}$, \\ Mayila Yusupu ${ }^{3,4}$, Hugh Blair ${ }^{5}$, Paul Kenyon ${ }^{5}$, Stephen Morris ${ }^{5} \mathbb{D}$, Wenrong Li ${ }^{3,4, *}$ and \\ Mingjun Liu ${ }^{3,4, *}$ \\ 1 College of Life Science and Technology, Xinjiang University, Urmuqi 830000, China; lvxuefeng@xjaas.net \\ 2 Institute of Animal Husbandry Quality Standards, Xinjiang Academy of Animal Sciences, \\ Urmuqi 830000, China \\ 3 Key Laboratory of Genetics, Breeding \& Reproduction of Grass-Feeding Livestock, Ministry of Agriculture, \\ Urmuqi 830000, China; chenlei0991@126.com (L.C.); Hesangang3@163.com (S.H.); \\ chenxi_4000@163.com (C.L.); hanbin0991@163.com (B.H.); 1xfxjxm@outlook.com (Z.L.); \\ mayila09910@163.com (M.Y.) \\ 4 Key Laboratory of Animal Biotechnology of Xinjiang Institute of Animal Biotechnology, Xinjiang Academy \\ of Animal Science, Urmuqi 830000, China \\ 5 International Sheep Research Centre, School of Agriculture and Environment, Massey University, \\ Palmerston North 4442, New Zealand; h.blair@massey.ac.nz (H.B.); p.r.kenyon@massey.ac.nz (P.K.); \\ s.t.morris@massey.ac.nz (S.M.) \\ * Correspondence: liwenrong@xjaas.net (W.L.); liumingjun@xjaas.net (M.L.) \\ + These authors contributed equally to this work.
}

Received: 11 May 2020; Accepted: 17 June 2020; Published: 19 June 2020

Simple Summary: The morphogenesis hair follicles begins in the embryonic stage. The first follicles formed are the primary wool follicles (PF), followed by secondary wool follicles (SF), and then secondary-derived follicles that branch from the SF in Merino sheep. The morphogenesis and development of SF in sheep determine wool yield and quality. At present, the mechanism of SF development and branching is still unclear. In this study, hair follicle morphogenesis and development were inhibited through nutritional restrictions during pregnancy, and transcriptome sequencing was performed to screen for differentially expressed genes related to the SF development in the fetus. Our findings are helpful to understand the mechanisms of SF development and branching of Merino sheep.

\begin{abstract}
The high concentration of secondary branched wool follicles is a distinctive feature of the Merino sheep. At present, the molecular control of the development and branching of secondary wool follicles (SF) remains elusive. To reveal the potential genes associated with the development of hair follicles, we investigated the characteristics of prenatal and postnatal development of wool follicles, and the transcriptional expression profile in fetuses/lambs from dams under either maternal maintenance or sub-maintenance (75\% maintenance) nutrition. The density of SF and the ratio of SF to primary wool follicles $(\mathrm{PF})$ were reduced $(p<0.05)$ in fetuses from day 105 to 135 of gestation under sub-maintenance nutrition. Differentially expressed genes were enriched in the binding, single-organism process, cellular process, cell and cell part Gene Ontology (GO) functional categories and metabolism, apoptosis, and ribosome pathways. Four candidate genes, SFRP4, PITX1, BAMBI, and KRT16, which were involved in secondary wool follicles branching and development, were identified. Our results indicate that nutritional intervention imposed on pregnant ewes by short-term sub-maintenance nutrition could provide a strategy for the study of wool follicle development. Overall insight into the global gene expression associated with SF development can be used to investigate the underlying mechanisms of SF branching in Merino sheep.
\end{abstract}


Keywords: Chinese Merino sheep; wool follicle; nutritional restriction; transcriptome

\section{Introduction}

In sheep, wool follicle morphogenesis begins in the embryonic stage and requires coordination and communication of multiple signals to regulate their growth [1]. The Chinese Merino sheep was developed in 1985 and is characterized by its large body size, high density, and white wool with a fineness (diameter) between 18 and $25 \mu \mathrm{m}$ [2]. Wool yield is the most important economic driver for fine wool sheep, which is affected by many factors [3]. The density of wool follicles affects wool yield while the diameter determines the wool's value. In follicle development, the first follicle formed is the primary wool follicle (PF) which occurs at approximately day 70 of gestation, followed by secondary wool follicles (SF), starting at approximately day 85 of gestation. The secondary-derived wool follicles begin to be initiated at approximately day 105 of gestation [1]. Based on previous studies, nutrition is crucial for SF morphogenesis and development, and malnutrition directly affects the density of wool follicles [4]. Moderate nutritional restriction in ewes during days 85-135 of gestation affects the morphogenesis and branching of SF, resulting in slower SF maturation and a reduced proportion of secondary to primary wool follicles $(\mathrm{S} / \mathrm{P})[5]$.

At present, many candidate genes related with economically important traits of sheep have been discussed [6]. However, the genes related to wool quality and yield still need further study. The morphogenesis and development of wool follicles involve a complex process that are dependent on a number of genes and signaling pathways [7]. The morphogenesis of wool follicles in Merino sheep is similar to those of cashmere goats [8]. Studies on wool follicle cycling and SF development in cashmere goats have identified a large number of genes related to follicle and fiber development in which the mechanism of SF development has been elucidated using high throughput RNA sequencing approach [9-12]. However, the morphogenesis and genes or signaling pathways that regulate fetal SF development and branching in Merino sheep require further investigation. To study the spatial-temporal complexity of follicle development, we collected fetal and lamb skin tissue at five different time points during pregnancy under the conditions of regular feeding and restricted feeding. This experimental design aimed to investigate the PF and SF formation and its characteristics and to obtain global gene expression profiles of differentially expressed genes (DEGs). The purpose of this study was to identify possible genes involved in the development of SF and secondary-derived wool follicles and to evaluate the effects of sub-nutrition at different gestational moments at wool follicle and development. This knowledge can be used to understand the molecular mechanisms involved in SF branching in fine wool sheep.

\section{Material and Methods}

\subsection{Study Design}

Chinese Merino ewes weighing 40-60 kg with wool fiber thinness between 20 and $23 \mu \mathrm{m}$ were selected from a breeding farm in Urumqi, located in the Xinjiang Province of China. Estrous cycles were synchronized using vaginal medroxyprogesterone acetate-impregnated sponges, which were removed $48 \mathrm{~h}$ before artificial insemination. Semen from 3 different Chinese Merino rams with wool fiber diameter 20-23 $\mu \mathrm{m}$ were used for artificial insemination. Thirty days after artificial insemination, ewe pregnancy was detected by a B-ultrasound scan. Based on previous studies of hair follicles morphogenesis and development [1,13], the effects of nutrition in five time periods were examined. These included days 55 to 85,85 to 105, and 105 to 135 of gestation, day 135 of gestation to 7 days after birth, and 7 to 35 days after birth. During each period ewes were offered either sub-maintenance or maintenance feeding with 3 ewes in each subgroup (Figure 1). The study design included a total of 30 ewes and the number of groups are shown in Figure 1. The impacts of dam postnatal nutrition were 
also examined, because it is known that secondary wool follicle development and maturation can take up to 5 weeks after birth [14] and the lamb is dependent on its dams milk during that period.

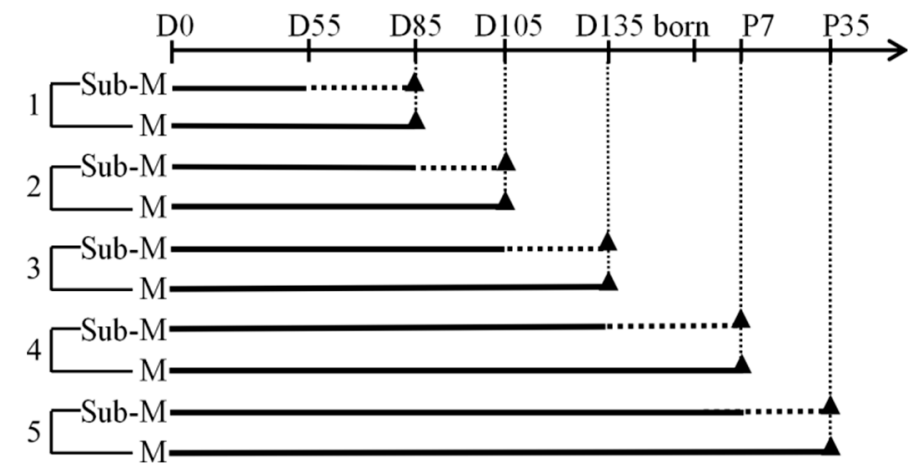

Figure 1. Timelines of the maternal feed restriction experiment. The experiments were divided into five groups (1-5), each group included sub-maintenance (Sub-M) nutrition ewes ( $n=3)$ and maintenance (M) nutrition ewes $(n=3)$. The timeline at the top of the diagram represents the gestational period. D0: day 0 of gestation; D55: days 55 of gestation; D85: days 85 of gestation; D105: days 105 of gestation; D135: days 135 of gestation; P7: postnatal day 7; P7: postnatal day 35. The solid line represents the maintenance nutrition feeding period, the dotted line represents the Sub-maintenance nutrition feeding period. Triangle represents sampling time points. The vertical dotted lines are used to align the sampling time points of the Sub-M and M.

\subsection{Animal Feeding}

All ewes were subjected to identical feeding regimens. The nutritional requirements for M group were based on the feeding standard of the New Zealand Society of Animal Production (NZSAP) (Table S1). Ewes were fed a mixed pellet that contained $8.92 \mathrm{MJ}$ metabolizable energy per $\mathrm{kg}$. The nutrient composition of the pellet consisted of $94 \%$ dry matter, $13.55 \%$ crude protein, $2.25 \%$ crude fat, $28.41 \%$ neutral cellulase, $8.89 \%$ acid cellulase, $6.26 \%$ crude ash, $0.48 \%$ calcium, and $0.49 \%$ phosphorus. Daily feed intake of Sub-M group ewes was 75\% of the M group. The daily feed intake in each group is shown in Table S1. The animals used in this study were managed at the Research Base of Sheep Breeding of Xinjiang Academy of Animal Science, located at Urumqi city in Xinjiang province of China. Surgeries were performed under strict aseptic conditions and to minimize potential animal welfare effects. All animal handling procedures were carried out in strict accordance with approved guidelines of the Institutional Animal Care of Xinjiang Academy of Animal Science (No:20180603001).

\subsection{Biochemical Examination of Blood}

To determine the effect of sub-maintenance nutrition on the ewes and fetal development, ewe blood biochemical indexes were assayed every 7 days after start of each nutritional restriction. Blood samples were collected from the jugular vein and placed in centrifuge tubes. Centrifugation was performed at $3000 \mathrm{rpm}$ for $10 \mathrm{~min}$ and the serum was collected and analyzed by an automated biochemistry analyzer (Olympus AU 400, Olympus-diagnostic, Hamburg, Germany). The analyzed parameters included the following: Total protein (TP), Albumin (ALB), Alanine aminotransferase (ALT), Aspartate aminotransferase (AST), urea nitrogen (BUN), blood glucose (GLU), triglyceride (TG), high-density lipoprotein (HDLC), low-density lipoprotein (LDLC), Free fatty acids (FFA).

\subsection{Histological Analysis of Wool Follicles}

At the end of each feed restriction period, fetuses were removed by caesarean section. The body length, chest circumference, abdominal circumference, body weight, and the weight of internal organs were measured. Fetus skin samples were collected from the right mid-side of the fetuses, and then placed in centrifuge tubes containing $4 \%$ paraformaldehyde solution. Alcohol concentrations of $50 \%$, 
$75 \%, 85 \%, 95 \%$, and $100 \%$ were used separately for $1 \mathrm{~h}$ tissue dehydration, followed by tissue clearing for $2 \mathrm{~h}$ using xylene and then submerging the samples in $56^{\circ} \mathrm{C}$ wax for $6 \mathrm{~h}$. The samples were then embedded in paraffin at $60^{\circ} \mathrm{C}$ and cut to $5 \mu \mathrm{m}$ diameters using a micro-slicing machine (Leica RM2235, Leica Biosystems Ltd., Shanghai, China). The tissue sections were stained using Hematoxylin-eosin (HE), fixed with neutral resin, and photographed and observed under microscope. Primary and secondary wool follicle density and the S/P were measured. The PF and SF were differentiated through the presence/absence of sweat and sebaceous glands. Three independent slices were measured for each sample, with at least 10 different microscopic fields examined for each slice. The data were expressed the mean \pm standard deviations (SD). A Tukey's test following one-way ANOVA in SPSS 19.0 (IBM, NY, USA) was used for a significance analysis.

\subsection{Transcriptome Sequencing}

Skin samples were collected from all treatment groups and immediately frozen in liquid nitrogen. Following histological analysis of wool follicles, a significant difference in SF and S/P between $\mathrm{M}$ and Sub-M groups at days 135 of gestation were identified. Hence, 3 independent skin samples from day105 of gestation for the M group (D105), day135 of gestation for the M group (D135) and D135 of gestation for the Sub-M group (DR135) were analyzed. Total RNA was isolated using Trizol Reagent (Invitrogen, CA, USA), according to the manufacturer's instructions. RNA libraries for each skin sample were constructed. Oligo (dTs) were used to isolate poly (A) mRNA. The mRNA was fragmented and reversed transcribed using the random primers. Second-strand cDNAs were synthesized using RNase $\mathrm{H}$ and DNA polymerase I. Double-strand cDNAs were then purified using a QIAQuick PCR purification kit (QIAGEN, Dusseldorf, Germany). The expected fragments were purified via agarose gel-electrophoresis after PCR amplification. Finally, amplified fragments were sequenced using the Illumina HiSeqTM 2000 instrument (Novogene, Beijing, China), according to the manufacturer's specification.

\subsection{Data Processing}

Raw reads of all samples were pre-processed via the removal of adaptors with more than $5 \%$ unknown nucleotides. Low-quality reads, which are the percentage of low-quality bases, were also removed. Clean reads were aligned against the reference genes Ovis aries (Oar_v3.1) with SOAPaligner/SOAP2 and were annotated. Mismatch of no more than two bases was allowed for alignment to obtain uniquely mapped reads. The fragments per kilobase of exon model per million reads mapped (FPKM) was used to normalize the transcripts and estimate the expressed gene levels. Differentially expressed genes (DEGs) were identified between the two groups using the DESeq2 R package (version 1.8.1) (https://bioconductor.org/packages/release/bioc/vignettes/DESeq2/inst/doc/ DESeq2.html). The Benjamini and Hochberg false discovery rate (FDR) was applied for $p$-value correction in multiple hypotheses. In this study, an adjusted $p$-value(padj) of $<0.05$ and $\mid \log 2$ (fold change) $\mid>0.5$ were set as the criteria to filter the DEGs.

\subsection{GO and KEGG Pathway Analysis}

GO (gene ontology) annotation was analyzed using Blast2GO software (version 2.3.5, Biobam, Valencia, Spain) (https://www.geneontology.org). The enrichment analysis of KEGG (Kyoto Encyclopedia of Genes and Genomes) pathways was implemented using KOBAS (version 3.0) (http://kobas.cbi.pku.edu.cn/index.php), padj $<0.05$ were considered significantly enriched. The gene interaction network was drawn using Ingenuity Pathway Analysis (IPA: http://www.ingenuity. com) software.

\subsection{Validation of Transcriptome Data Using qRT-PCR}

To validate RNA-Seq sequencing data, 8 DEGs were selected for qRT-PCR analysis. Primers used in the qRT-PCR were designed using primer 5 (Table S3). The PCR conditions were carried out as 
follows: initial denaturation at $95{ }^{\circ} \mathrm{C}$ for $10 \mathrm{~min}$, followed by 40 cycles of $5 \mathrm{~s}$ denaturationat $95{ }^{\circ} \mathrm{C}$, annealing at $60{ }^{\circ} \mathrm{C}$ for $30 \mathrm{~s}$ and extension at $72{ }^{\circ} \mathrm{C}$ for $20 \mathrm{~s}$. The qRT-PCR reaction of each gene in each sample was repeated three times, the relative expression of each gene was normalized to GAPDH and calculated using the $2^{-\Delta \Delta \mathrm{Ct}}$ method, and final results are shown as means $\pm \mathrm{SD}$.

\section{Results}

\subsection{Changes in Blood Biochemical Indexes}

Most blood biochemical indexes showed no difference between pregnant ewes under $\mathrm{M}$ and Sub-M nutrition. However, HDLC was higher $(p<0.05)$ in M than in Sub-M on days 14, 21, and 28. Blood glucose level was lower $(p<0.05)$ for M compared to Sub-M treatments on day 21. NEFA was lower $(p<0.05)$ in M than Sub-M treatments on day 28. No other differences were observed $(p>0.05)$ (Table S2).

\subsection{Morphogenesis and Development of Wool Follicles}

There was no difference in fetus weight, body size, and organ development between nutritional groups. However, kidney weight was significantly $(p<0.05)$ lower in the Sub-M treatment compared to the M group at day 135 of gestation (Table S4). On day 85 of gestation, PF with a hairy bud-like structure surrounded by sebaceous glands were observed (Figure 2). The arrangement of wool follicles appeared regular and the morphology of SF featured an aggregation of dermal cells into clumps. On day 105 of gestation, dicotyledonous sebaceous glands were found around the PF, while the SF bud had extended downward and had penetrated deep into the dermis. With the increase of the diameter of the dermal papillae, the sebaceous glands began to form, although the hair shafts were not observed in PF and SF at this stage. On day 135 of gestation, completed sebaceous and sweat glands were formed around the PF. Sebaceous glands with bilobed structures were prominent and sweat glands were visible in lumen structures. SF had no sweat glands but contained sebaceous glands. In contrast to SF, the PF grew some hair shafts. On day 7 after birth, SF were still growing with some of them being devoid of hair shafts. In both $\mathrm{M}$ and Sub-M groups, most of the wool follicles grew hair shafts by 35 days after birth (Figure 2).
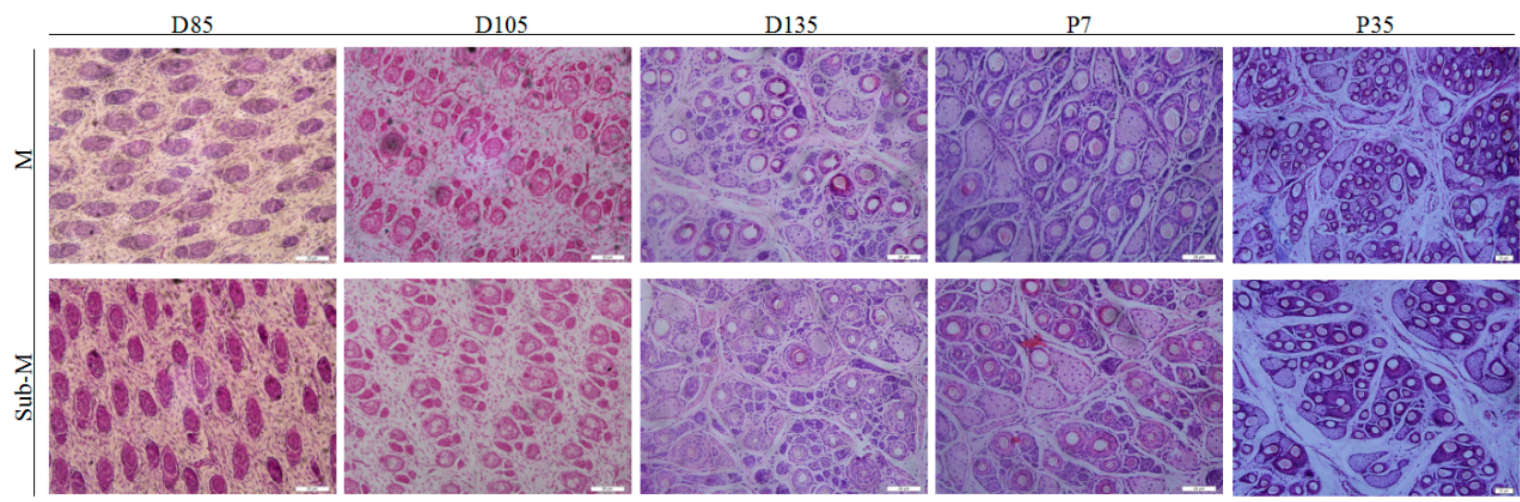

Figure 2. Morphogenesis and development of wool follicles in pregnancy maintenance and nutritional restriction group of Merino ewes. Lateral skin from fetal and lamb bodies was obtained; the deparaffinized sections were stained with H\&E. Scale bars: $20 \mu \mathrm{m}$. The timeline at the top of the diagram represents the gestational period: D85, 85 days of gestation; D105, 105 days of gestation; D135, 135 days of gestation; P7, 7 days after birth; and P35, 35 days after birth.

\subsection{The Density and S/P Ratios of Wool Follicles}

The density of PF, SF, and S/P ratios between M samples and Sub-M samples at days 85 and 105 of gestation showed no significant differences $(p>0.05)$. The SF density, total density and S/P ratio of Sub-M samples were lower $(p<0.05)$ than those of the M group at days 135 of gestation. There was no 
difference $(p>0.05)$ in the densities of $\mathrm{PF}$ and $\mathrm{SF}$, or S/P ratios between the $\mathrm{M}$ and Sub-M at 7 days after birth. The $\mathrm{S} / \mathrm{P}$ ratios of $\mathrm{M}$ samples were higher $(p>0.05)$ than those of the Sub-M at day 35 after birth (Table 1).

Table 1. Wool follicles density and S/P ratios of M and Sub-M group at different gestation stages.

\begin{tabular}{|c|c|c|c|c|c|c|}
\hline Time & Groups & Number & $\begin{array}{l}\text { Primary Wool } \\
\text { Follicles } \\
\text { Density }\left(\mathrm{mm}^{2}\right)\end{array}$ & $\begin{array}{c}\text { Secondary Wool } \\
\text { Follicles } \\
\text { Density }\left(\mathrm{mm}^{2}\right)\end{array}$ & $\begin{array}{l}\text { Total Density } \\
\qquad\left(\mathrm{mm}^{2}\right)\end{array}$ & $\begin{array}{c}\text { Proportion of } \\
\text { Secondary to } \\
\text { Primary Wool } \\
\text { Follicles }\end{array}$ \\
\hline \multirow{2}{*}{ D85 } & $M$ & 3 & $84.15 \pm 12.72$ & $23.14 \pm 14.10$ & $107.30 \pm 2.62$ & $0.30 \pm 0.13$ \\
\hline & Sub-M & 3 & $82.83 \pm 34.18$ & $30.66 \pm 9.11$ & $113.49 \pm 43.24$ & $0.31 \pm 0.07$ \\
\hline \multirow{2}{*}{ D105 } & $\mathrm{M}$ & 3 & $71.75 \pm 9.84$ & $144.89 \pm 34.17$ & $216.64 \pm 40.31$ & $2.02 \pm 0.41$ \\
\hline & Sub-M & 3 & $72.61 \pm 9.25$ & $114.14 \pm 22.83$ & $187.05 \pm 14.01$ & $1.60 \pm 0.50$ \\
\hline \multirow{2}{*}{ D135 } & $M$ & 3 & $28.56 \pm 6.59$ & $201.68 \pm 33.89^{a}$ & $230.24 \pm 39.97^{a}$ & $7.19 \pm 0.71^{a}$ \\
\hline & Sub-M & 3 & $26.99 \pm 3.72$ & $139.34 \pm 17.30^{b}$ & $166.33 \pm 19.86^{b}$ & $5.25 \pm 0.60^{b}$ \\
\hline \multirow{2}{*}{ P7 } & M & 3 & $13.29 \pm 1.71$ & $113.75 \pm 22.98$ & $127.04 \pm 24.69$ & $8.62 \pm 0.75$ \\
\hline & Sub-M & 3 & $16.50 \pm 3.19$ & $154.50 \pm 11.31$ & $171.00 \pm 14.50$ & $9.59 \pm 1.30$ \\
\hline \multirow{2}{*}{ P35 } & $\mathrm{M}$ & 3 & $6.67 \pm 0.46$ & $69.25 \pm 11.19$ & $91.53 \pm 8.90$ & $10.34 \pm 1.03$ \\
\hline & Sub-M & 3 & $9.88 \pm 1.94$ & $95.95 \pm 24.67$ & $105.82 \pm 26.62$ & $9.79 \pm 0.50$ \\
\hline
\end{tabular}

Data are means $\pm \mathrm{SD},{ }^{\mathrm{a}, \mathrm{b}}$ Within column, different superscript letter indicates differences $(p<0.05)$ between $\mathrm{M}$ and Sub-M group at the same gestation stages.

\subsection{DEGs Analysis}

The transcriptional profiles of D105, D135, and DR135 samples revealed a total of 20,062, 20,653, and 20,846 transcripts, respectively. A total of 2164, 2739, and 692 DEGs in D135 vs. D105, DR135 vs. D105, and DR135 vs. D135, respectively, were obtained (Tables S5-S7). Co-expressed DEGs, specific DEGs and comparisons of D135 vs. D105, DR135 vs. D105, and DR135 vs. D135 are shown in Figure 3 a. Up-regulated and down-regulated genes for comparisons between D135 vs. D105, DR135 vs. D105 and DR135 vs. D135 are shown in Figure 3b. Nutritional restriction was associated with changes in the number of DEGs.
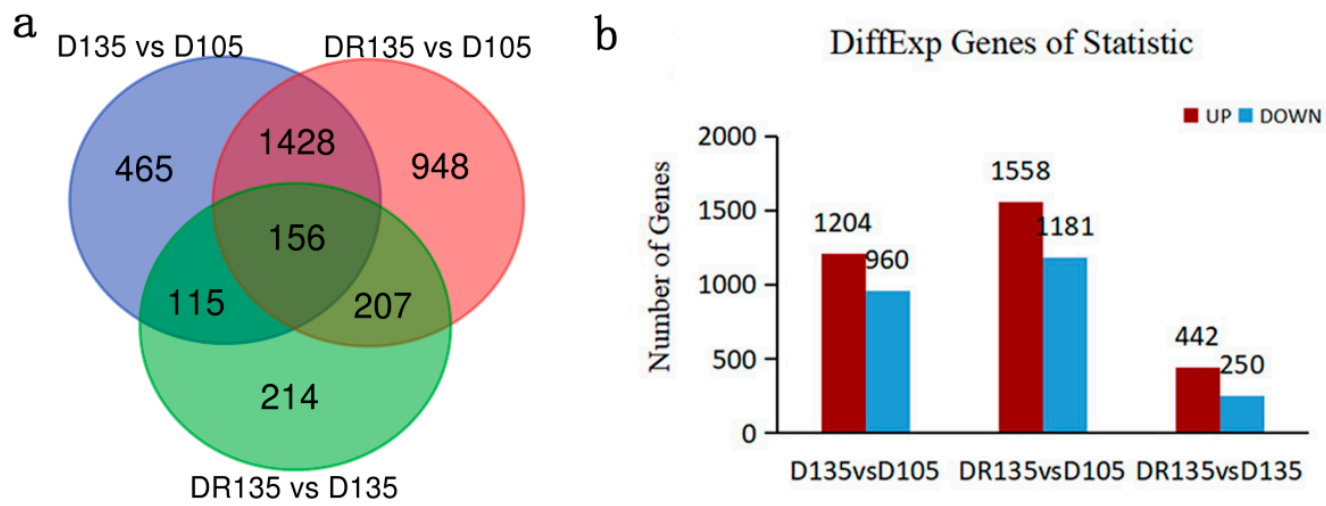

Figure 3. Summary of DEGs. (a) Venn diagram illustrating the overlaps of DEGs between D135 vs. D105, DR135 vs. D105 and DR135 vs. D135. (b) Number of up-regulated and down-regulated expression genes between D135 vs. D105, DR135 vs. D105, and DR135 vs. D135. The x-axis represents the comparison scheme between the indicated different time points. The y-axis represents the corresponding number of DEGs. The red columns represent up-regulated expression genes and blue columns represent down-regulated expression genes.

\subsection{GO and KEGG Analysis of the DEGs}

To understand the processes driving the changes in histomorphology of wool follicles after feed restrictions, GO terms analysis of DEGs was carried out. The DEGs of D135 vs. D105, DR135 vs. D105, and DR135 vs. D135 were annotated in 54, 54, and 46 functional categories, respectively 
(Figure 4). For biological processes, the top three functional categories were single-organism process, cellular process, and metabolic process, all three are involved in cell proliferation and tissue or organ development. It also includes most of the known genes related to wool follicle development. In terms of cellular components, the top three functional categories were cell, cell part, and organelle, these include genes that are related to cell structure, junction, and communication. For molecular function, most genes were classified into binding, which mainly involves protein binding, ion binding, carbohydrate derivative binding, and sulfide binding-related genes.

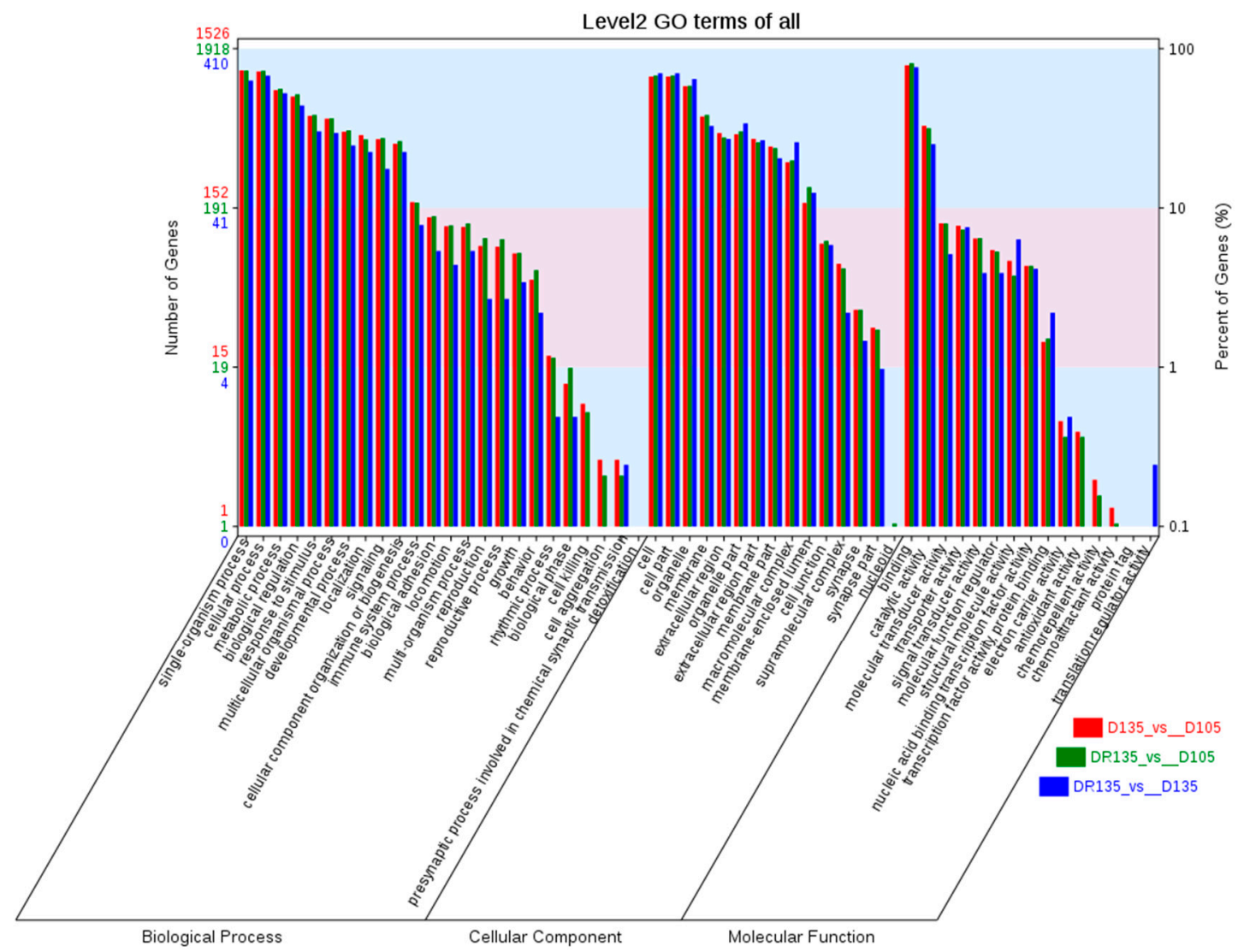

Figure 4. A Gene Ontology (GO) functional enrichment analysis of DEGs. The x-axis shows the three categories of Gene Ontology; the y-axis shows the number of genes (left) and percent of genes (right). The red bars represent DEGs number in D135 vs. D105. The green bars represent DEGs number inDR135 vs. D105 and Blue bars represent DEGs number inDR135 vs. D135.

DEGs were also analyzed by KEGG enrichment pathway. In D135 vs. D105, Protein digestion and absorption, tight junction and cell adhesion molecules (CAMs) pathways were the most abundant pathways (padj < 0.05) in the top 20 KEGG pathways (Figure 5a). Many genes related to hair follicle development, including CAV3, COL6A, COL21A, ITGA9, VDR, MAP3K7, TGF- $\beta 2$, PLCB, WNT2, and WNT16, were identified in these pathways. In DR135 vs. D105, apoptosis and Mucin type O-glycan biosynthesis were the most abundant pathways in the top 20 KEGG pathways (Figure 5b). In addition, the pathways related to hair follicle development, including cell adhesion molecules (CAMs), ECM-receptor interaction and tight junction, were also found in the top 20 pathways. In DR135 vs. D135, ribosome and oxidative phosphorylation were the most significantly enriched pathways (Figure 5c). 

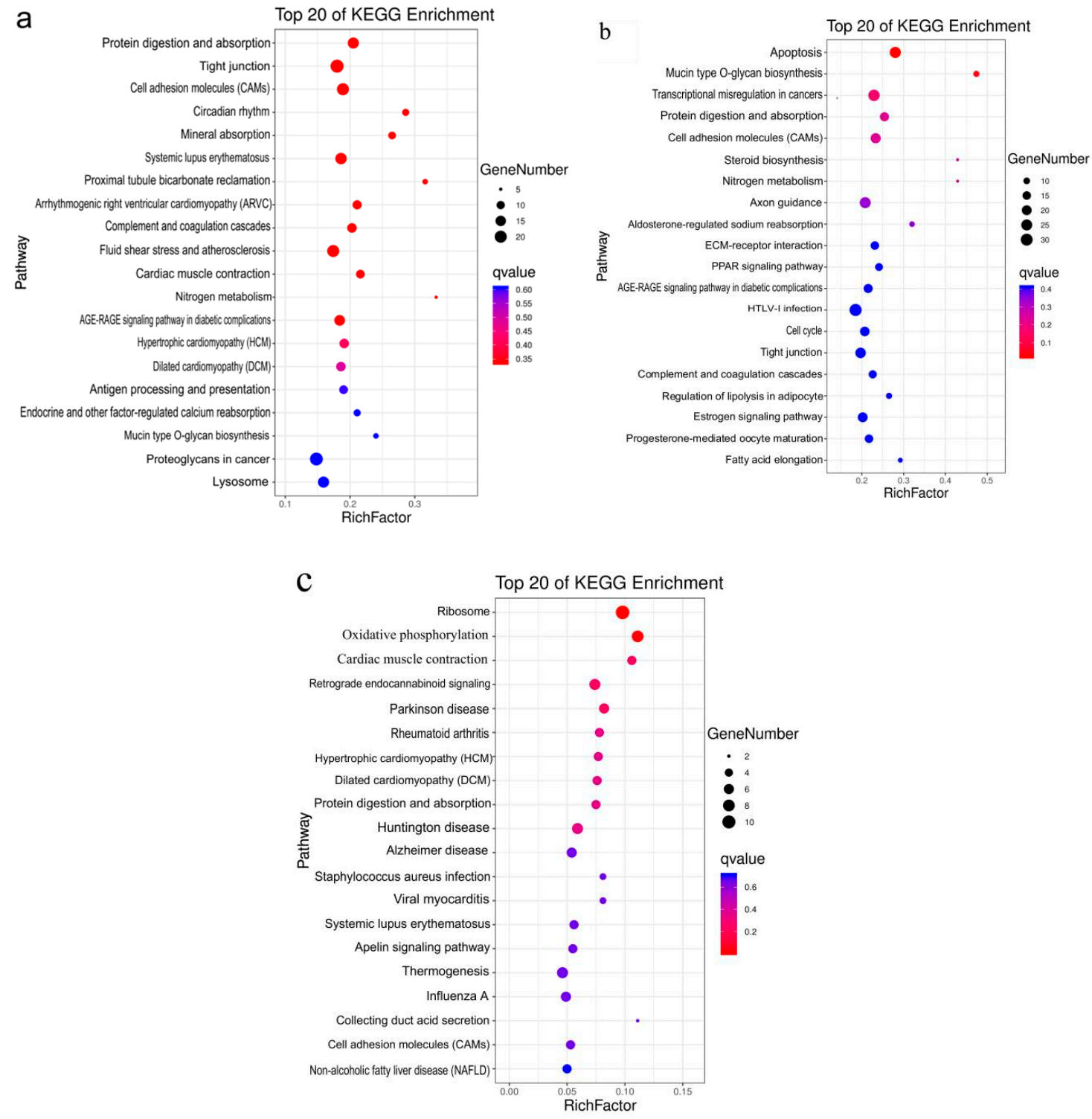

Figure 5. Top 20 KEGG enrichment pathways of DEGs. (a) KEGG enrichment of DEGs in D135 vs. D105. (b) KEGG enrichment of DEGs in DR135 vs. D105. (c) KEGG enrichment of DEGs in DR135 vs. D135. The $x$-axis represents rich factor and the $y$-axis represents the KEGG pathways.

\subsection{Changes in Signaling Pathway Genes Related to Wool Follicle Development}

The Wnt and TGF- $\beta$ /BMP signaling pathways are closely related to wool follicle development. To understand the changes in genes related to these two signaling pathways, key genes for wool follicle development were screened. A total of $20 \mathrm{Wnt}$ signaling pathway-related genes and 23 TGF- $\beta$ /BMP signaling pathway-related genes were identified among DEGs. The expression patterns of the two signaling pathway-related genes for D105, D135, and DR135 samples were analyzed using a cluster heat map. Among the $20 \mathrm{Wnt}$ pathway-related genes, 12 genes were down-regulated and 8 were up-regulated from day 105 to 135 of gestation in M samples (Figure 6a). The expressions of WNT2, WNT5A, and WNT16 were down regulated at D135 when compared to the D105. DKK and SFRP are inhibitors of Wnt signaling. The analyses of these inhibitors in D135 showed that the expressions of $D K K 1$, SFRP2, and SFRP4 were down-regulated, whereas DKK2, SFRP1, and SFRP5 were up-regulated. In the Sub-M group (DR135), the gene expression profile mostly displayed a similar trend to the D135 group. However, the expression of FRAT2 was down-regulated in D135 but up-regulated in DR135. 
The expression of PLCB4 and SFRP1 showed an up-regulated trend in D135 and a down-regulated trend in DR135 in comparison with D105.

a

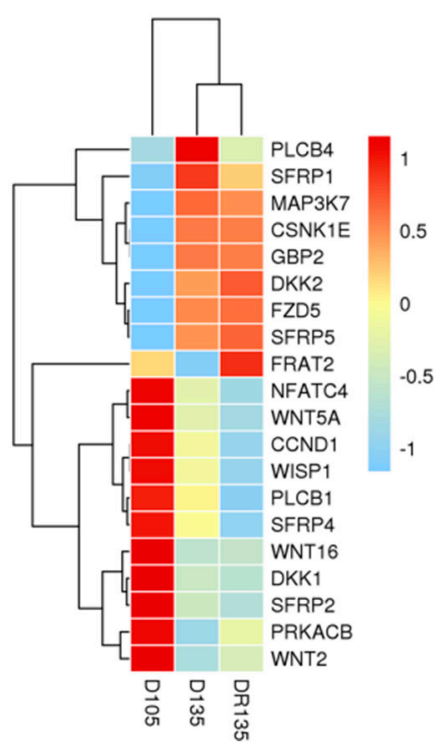

b

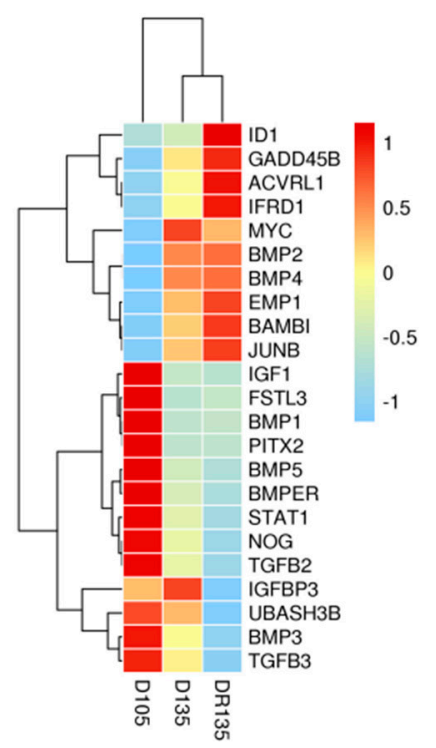

Figure 6. Heat map of the hierarchical clustering of DEGs. (a) Clustering heat map of Wnt signal pathway correlated genes. (b) Clustering heat map of TGF- $\beta$ /BMP signaling pathway genes. Each row represents the DEGs, and each column represents samples at a time point. Heat map uses FPKM value and then standardizes. Blue and red gradients indicate a decrease and increase in transcript abundance, respectively. The line on the left represents gene cluster, and the line on the top represents sample cluster.

Among the TGF-beta/BMP pathway-related genes, 12 genes were down-regulated and 11 were up-regulated from days 105 to 135 of gestation in M group (Figure $6 \mathrm{~b}$ ). The expressions of $M Y C, B M P 2$, $B M P 4$ and $B A M B I$ were significantly up-regulated, while the expression of IGF1, PITX2, BMP5, NOG, $T G F-\beta 2, B M P 3$ and TGF- $\beta 3$ were significantly down-regulated. Compared with D135, the expression of these genes was further up-regulated or down-regulated in DR135. The expression of the BAMBI was greatly up regulated at DR135 vs. D135. The expression of the IGFBP3 was up-regulated at D135 vs. D105 and down-regulated at DR135 vs. D105.

\subsection{Identification of Key Genes for Secondary Follicles Development and Branching}

The branching of secondary follicles (SF) in fine wool sheep occurs between 105 and 135 days of gestation. SF emerge in the neck of developing secondary original follicles that leads to the formation of secondary-derived follicles. The functional genes involved in this process and their mechanisms are currently unclear. To further investigate the mechanism of secondary follicle development in fine-wool sheep, genes related to wool follicle development, epithelial cell proliferation and regulation, keratin differentiation, and branching morphology were screened for DEGs. (Table 2). We found significant differences in the expression of BMP5, CAV3, DNASE1L2, FOXN1, FOXA1, SLURP1, MMP12, SFRP2, SFPR4, PITX1, PITX2, KRT16, BAMBI, and SIX4 from 105 to 135 days of gestation. Meanwhile, the expression of SFRP4, PITX1, and KRT16 was significantly different in DR135 vs. D135, wherein the expression of PITX1 was up-regulated and the expressions of SFRP4 and KRT16 were down-regulated. These findings indicate that the changes in expression levels may be related to the development and branching of SF. 
Table 2. Potential key genes that are differentially expressed related to SF development.

\begin{tabular}{|c|c|c|c|c|}
\hline \multirow{2}{*}{ Gene } & \multirow{2}{*}{ Description } & \multicolumn{3}{|c|}{ log2 (Fold Change) } \\
\hline & & D135 vs. D105 & DR135 vs. D105 & DR135 vs. D135 \\
\hline \multicolumn{5}{|c|}{ Wnt related } \\
\hline SFRP2 & Secreted Frizzled Related Protein 2 & -2.40341 & -2.79309 & -0.38968 \\
\hline SFRP4 & Secreted Frizzled Related Protein 4 & -1.07911 & -2.10080 & -1.02169 \\
\hline SFRP5 & Secreted Frizzled Related Protein5 & 1.45166 & 1.62546 & 0.17380 \\
\hline WNT5A & Wnt Family Member 5A & -0.37882 & -0.51687 & -0.13805 \\
\hline WNT2 & Wnt Family Member 2 & -1.14818 & -0.92800 & 0.22018 \\
\hline$D K K 1$ & Dickkopf-Related Protein 1 & -1.44226 & -1.56450 & -0.12224 \\
\hline CCND1 & Cyclin D1 & -0.40895 & -0.70606 & -0.29711 \\
\hline CSNK1E & Casein Kinase 1 Epsilon & 2.69495 & 2.67435 & -0.02060 \\
\hline \multicolumn{5}{|c|}{ TGF- $\beta /$ BMP } \\
\hline$B M P 2$ & Bone Morphogenetic Protein 2 & 1.10571 & 1.19215 & 0.08644 \\
\hline$B M P 3$ & Bone Morphogenetic Protein 3 & -0.57071 & -1.11402 & -0.54331 \\
\hline$B M P 4$ & Bone Morphogenetic Protein 4 & 0.78090 & 0.83946 & 0.05856 \\
\hline BMP5 & Bone Morphogenetic Protein 5 & -2.48384 & -2.95107 & -0.46723 \\
\hline TGF- $\beta 2$ & Transforming Growth Factor Beta 2 & -0.73641 & -1.11444 & -0.37803 \\
\hline TGF- $\beta 3$ & Transforming Growth Factor Beta 3 & -0.33778 & -0.75208 & -0.41430 \\
\hline$B A M B I$ & BMP And Activin Membrane-Bound Inhibitor & 1.05036 & 1.55454 & 0.50418 \\
\hline$M Y C$ & $\begin{array}{l}\text { V-Myc Avian Myelocytomatosis Viral } \\
\text { Oncogene Homolog }\end{array}$ & 0.57669 & 0.42712 & -0.14958 \\
\hline \multicolumn{5}{|c|}{ Wool follicledevelopment and transcriptional regulator genes } \\
\hline PITX1 & Paired Like Homeodomain 1 & -2.93483 & -0.74502 & 2.18981 \\
\hline$C A V 3$ & Caveolin 3 & -3.14244 & -2.48249 & 0.65995 \\
\hline SIX4 & SIX Homeobox 4 & -2.38845 & -1.90240 & 0.48605 \\
\hline SLURP1 & Secreted LY6/PLAUR Domain Containing 1 & 4.00780 & 3.56769 & -0.44011 \\
\hline GATA3 & GATA Binding Protein 3 & 0.71200 & 0.40038 & -0.31162 \\
\hline$E D A R$ & Ectodysplasin A Receptor & -0.91182 & -1.05320 & -0.14137 \\
\hline FOXN1 & Forkhead Box N1 & 2.21443 & 1.85361 & -0.36083 \\
\hline HOXC13 & Homeobox C13 & 0.69009 & 0.73500 & 0.04492 \\
\hline LGR5 & $\begin{array}{l}\text { Leucine-Rich Repeat Containing G } \\
\text { Protein-Coupled Receptor } 5\end{array}$ & 0.85120 & 0.84652 & -0.00468 \\
\hline MSX2 & Msh Homeobox 2 & 0.36489 & 0.65509 & 0.29020 \\
\hline DNASE1L2 & Deoxyribonuclease 1 Like 2 & 1.55896 & 2.07115 & 0.51219 \\
\hline VDR & Vitamin D Receptor & 1.10678 & 0.95640 & -0.15039 \\
\hline IGFBP3 & Insulin-Like Growth Factor Binding Protein 3 & 0.17093 & -0.45424 & -0.62518 \\
\hline \multicolumn{5}{|c|}{ Branching morphology related genes } \\
\hline PITX2 & Paired Like Homeodomain 2 & -3.06724 & -3.39049 & -0.32325 \\
\hline MMP12 & Matrix Metallopeptidase 12 & 3.74146 & 3.73938 & -0.00208 \\
\hline IGF1 & Insulin-Like Growth Factor 1 & -0.71163 & -0.75344 & -0.04181 \\
\hline SOX9 & SRY-Box 9 & 0.95372 & 0.63590 & -0.31782 \\
\hline FOXA1 & Forkhead Box A1 & 2.24977 & 2.56852 & 0.31876 \\
\hline \multicolumn{5}{|c|}{ Keratin differentiation-related genes } \\
\hline KRT16 & Keratin 16 & 1.27650 & -1.44261 & -2.71911 \\
\hline KRTAP8-1 & Keratin Associated Protein 8-1 & 4.37327 & 4.93892 & 0.56566 \\
\hline KRT13 & Keratin 13 & -7.51172 & -6.59676 & 0.91496 \\
\hline KRT78 & Keratin 78 & -2.72411 & -2.20584 & 0.51827 \\
\hline
\end{tabular}

\subsection{Validation of DEGs by $q R T-P C R$}

To evaluate the validity of RNA-seq data, eight transcripts were selected for examination by qRT-PCR. The results showed that the expression patterns both qRT-PCR and transcriptome sequencing data were consistent for 8 genes (Figure S1), which demonstrated the reliability of transcription data.

\section{Discussion}

\subsection{Wool Follicle Morphogenesis and Development}

According to previous reports $[15,16]$, the appearance of PF in fine wool sheep begins at about 40 days of fetal life, whereas the SF appear from around 90 to 126 days. In this study, the PF appeared in the fetus at 55 day of gestation while the SF appeared at 85 day, both being similar to the previous studies. The S/P ratio increased rapidly between 105 and 135 days of gestation, revealing that this was a crucial period for SF development. Sub-M nutrition of the dam partially inhibited the development 
of SF and reduced the number of SF. The development of PF and SF densities were similar between the Sub-M and M groups. As the fetus gained weight, the skin area also increased and consequently the density of PF gradually decreased. PF densities decreased rapidly during days 105 to 135 of gestation. By contrast, the density of SF increased during this period, and in particular, the density of SF was the highest at 135 days of gestation in the $\mathrm{M}$ group. In contrast to the density parameter, the S/P ratio increased rapidly in late pregnancy in both treatment groups but became relatively stable after birth.

Nutrition of pregnant ewes has the potential to affect the establishment of the wool follicle population in the fetus [5]. In this study, there was no difference in the density of PF between the M and Sub-M groups, indicating that Sub-M nutrition did not affect the morphogenesis and development of PF. This was consistent with the result of Schinckel and Short [17] who showed that PF density was independent of nutritional status of the fetus. There was no difference in SF density between Sub-M and M groups at days 85 and 105 of gestation. It is possible that the level of sub-nutrition was not sufficient to change wool follicle development. Between days 105 and 135 of gestation, SF branching occurred and wool follicle density in the Sub-M group was lower than that of the M group at day135 of gestation. This reduction may be due to the decrease and delay of SF branching caused by lower dam nutrition, which also resulted in a decrease in the $\mathrm{S} / \mathrm{P}$ ratio, which is consistent with previous findings [18,19]. In our study, the S/P ratio of the Sub-M group was $13 \%$ lower than that of $M$ group, which is consistent with Schinckel and Short [16]. However, there was no difference in S/P ratio after birth, suggesting that changes in SF population were delayed in Sub-M group. We also found that more SF did not grow hair shafts in the Sub-M group from day 135 of gestation to7 days after birth when compared to the $\mathrm{M}$ group. The maturation of SF is completed by approximately 12 weeks after birth [15]. Thus, it is likely the Sub-M treatment inhibited the development of wool follicles and delayed the development of the hair shafts.

Overall, our research showed that Sub-M nutrition led to a reduction in SF density by inhibiting the branching of SF during days 105 to 135 of gestation and provided an experimental model to study the molecular mechanisms of the formation of SF.

\subsection{Identification of Genes Related to Secondary Hair Follicle Branching}

We found that Sub-M nutrition hindered the development and branching of SF in fetuses alongside several changes in gene expression profiles. Menzies [20] reported that FGF10 and BMP4 were associated with secondary wool follicle branching in sheep. However, the genes related to secondary wool follicle branching are still uncertain. In this study, we provide an overall insight into global gene expression associated with Merino sheep wool follicle development and branching.

Wnt and TGF- $\beta$ /BMP signaling pathways play an important role in regulating wool follicles development [21-24]. We identified several genes related to the Wnt and TGF- $\beta /$ BMP signaling pathways in DEGs, such as WNT2, SFRP2, SFRP4, BAMBI, BMP2, and BMP5, which play an important role in hair follicle development. Using functional annotation and differential expression, we found there were differences in the expression of KRT16, NDASE1l2, CAV3, and PITX1 in DR135 and D135 fetuses. Using the same analyses, we identified 4 genes (SFRP4, PITX1, BAMBI, and KRT16) that might be essential in SF development and branching in Merino sheep follicles.

During the period of PF maturation, which is characterized by SF branching and hair shaft formation from $105 \mathrm{~d}$ to $135 \mathrm{~d}$ of gestation, both activation and inhibition of genes occurs in the Wnt and TGF- $\beta /$ BMP signaling pathways [25]. In contrast to D105, the expression at D135 of NOG was down-regulated and BAMBI up-regulated in TGF- $\beta /$ BMP signaling pathway. Meanwhile, in the Wnt signaling pathway, the expressions of DKK2, SFRP1, and SFRP 5 were up-regulated, while the expressions of DKK1, SFRP2, and SFRP4 were down-regulated at D135 vs. D105. These results suggest that the activation and inhibition modes of Wnt signaling occurred during wool follicle development. Inhibitors are involved in coordinating a regulatory balance during wool follicle development. The SFRP family seemed to play a more important role than DKK in wool follicle development during days 105-135 of gestation. SFRP4 inhibits the Wnt signaling pathway by phosphorylating $\beta$-catenin, thereby causing 
an arrest of cell division during the G2/M phase. SFRP4 inhibits wool follicle regeneration [26-28], and exogenous SFRP4 inhibits the proliferation of cultured human keratinocytes and promotes the apoptosis and differentiation of human keratinocytes in vitro [29,30]. The SFRP family increased Wnt activity at low concentrations and reduced Wnt activity at high concentrations [31]. The expression of SFRP4 was significantly down regulated in DR135 compared with D135. Here, we hypothesize that SFRP4 selectively inhibits different Wnt ligands and frizzled receptors, thus mediating the Wnt signaling pathway, and making it responsible for the decrease of secondary follicle branching and S/P ratio under dam Sub-M nutrition. However, the mechanism in regulating wool follicle development requires further investigation.

PITX1 belongs to the paired-bicoid homeobox protein family. It regulates embryonic development and differentiation and promotes cartilage and myogenesis in the hind limbs of mammals [32]. It also inhibits cell proliferation and migration and induces apoptosis [33,34]. SOX9 is a direct transcriptional target gene of PITX1. Vidal et al. [35] showed that SOX9, as one of the markers of stem cells, was expressed in the outer root sheath and bulge region of wool follicles. Overexpression of SOX9 can improve the cloning and proliferation of wool follicle outer root sheath cells [36]. Differentiation of stem cells may be necessary for SF branching. In this study, the expression of PITX1 was up regulated in DR135 compared to D135 samples, which may play an important role in keratinogenesis inhibition and wool follicle stem cell differentiation. It is also possible that these genes are related to SF branching.

TGF- $\beta$ /BMP is an indispensable signaling pathway in wool follicle development. TGF- $\beta 2$ regulates wool follicle development and growth cycles [37-39].The formation and development of wool follicles were inhibited, and the number of wool follicles was decreased in TGF- $\beta 2$ knockout mice [40]. BAMBI is an inhibitor of the TGF- $\beta$ family. It has a similar protein sequence to the TGF- $\beta$ I receptor and can bind to type II receptors to form a ligand-receptor complex. Due to $B A M B I$ lacking the serine/threonine kinase domain in the intracellular domain, it cannot be phosphorylated, consequently, it blocks the signal transduction of the TGF- $\beta$ family [41]. In our data, the expression of BAMBI was up-regulated, whereas the expression of TGF- $\beta 2$ and TGF- $\beta 3$ were down-regulated in DR135 vs. D135. It is suggested that $B A M B I$ may affect SF development through the TGF- $\beta$ pathway.

Keratin (KRTs) and Keratin-related protein (KRTAPs) are the most important component of wool fiber because they determine the structural characteristics of wool; they also play an important role in the development of wool follicles and wool fiber traits [42-45]. KRTs and KRTAPs gene expression profiles change with keratinocyte differentiation. The progression of hair follicle differentiation is characterized by the sequential activation of distinct sets of hair-specific KRTs and KRTAPs genes [46]. From 105 to 135 days of gestation, the expression of most KRTs and KRTAPs were up-regulated, except for four down-regulated KRTs, suggesting that the proliferation of hair keratinocytes was significantly active after 105 days of gestation. After feed restriction, seven KRTs and KRTAPs genes were down regulated. These results indicate that feed restriction can inhibit the proliferation of keratinocytes. Day 105-135 of gestation is the development and branching stage of SF in Merino sheep. The changes in KRTs and KRTAPs gene expressions were closely related to the development of SF. Expression of KRT16 was up-regulated in D135 vs. D105 but was down-regulated in DR135 vs. D105. We propose that KRT16 plays an important role in the development and branching of SF.

To determine the regulatory pathways of these 4 genes, we drafted the gene interaction networks using the IPA software (Figure 7). We found that BMP2, BMP4, SOX9, TGF- $\beta 2,3, M Y C$, and CCND1 were in key positions of the networks. Of note, the SFRP4 and PITX1 are located upstream of these genes. SFRP4 inhibits the Wnt signaling pathway by phosphorylating $\beta$-catenin, thereby blocking Wnt pathway transduction and regulating the transcription of target genes CCND1 and MYC. Wnt signaling is necessary for primary follicle morphogenesis and can promote the proliferation and differentiation of wool follicles stem cells. PITX1 is the downstream target gene of Wnt signaling pathway. On one hand, PITX1 regulates cell proliferation via the RAS pathway [47], while on the other hand, it regulates the differentiation of wool follicle stem cells by acting on FOXA1 and SOX9. SOX9 is essential for wool follicle regeneration. $B A M B I$ is an inhibitor of the members of the TGF- $\beta$ family and plays a role 
in wool follicle development. KRT16 is related to keratinocyte proliferation and also interacts with TGF- $\beta$. Hence, these four genes were closely related to wool follicle development and their functions are related to cell proliferation. In combination with the biological events of SF development during days 105 to 135 of gestation, we speculate that these genes play an important role in SF branching through the Wnt and TGF- $\beta$ pathways.

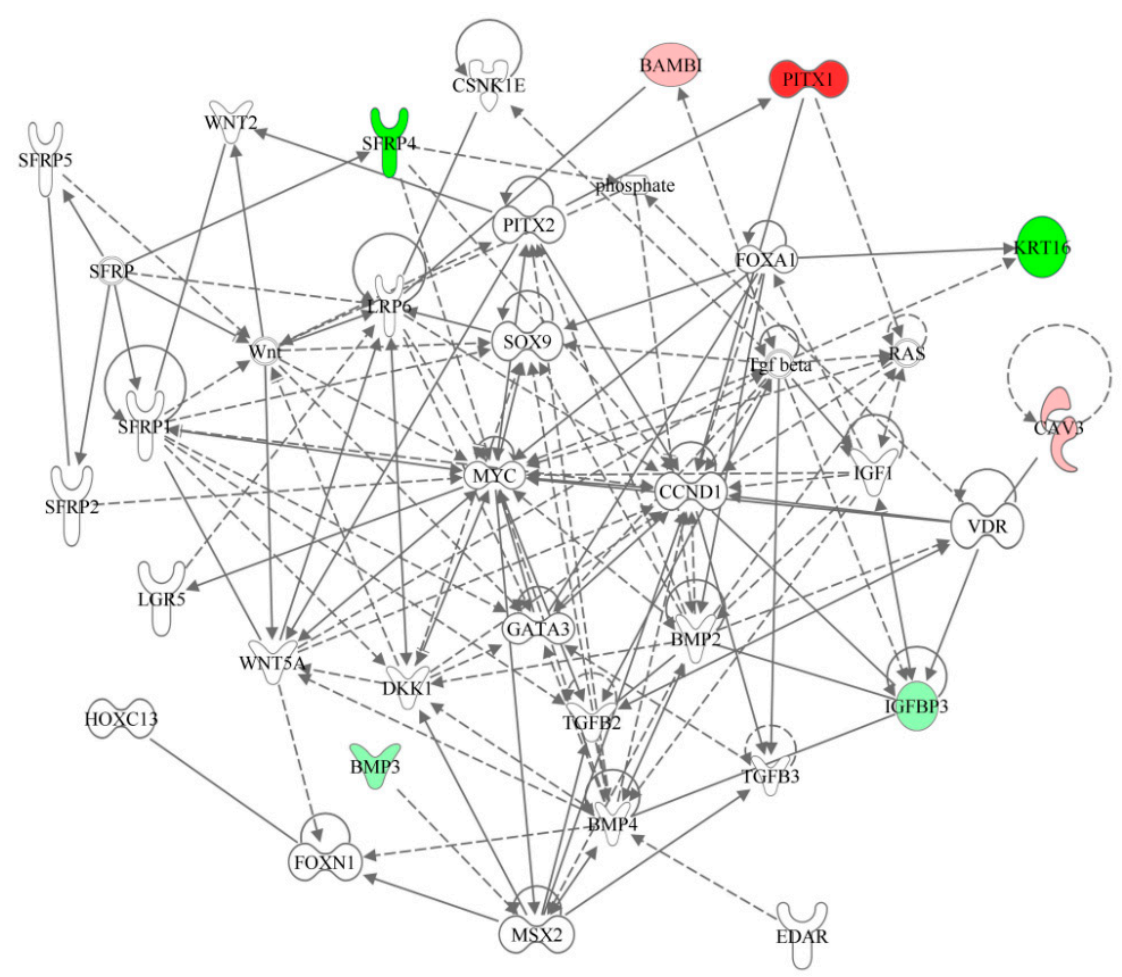

$$
\begin{aligned}
& \text { Complex } \\
& \text { Cytokine/Growth Factor } \\
& \text { Chemical/Toxicant } \\
& \text { Enzyme } \\
& \text { Group/Complex } \\
& \text { Growth factor } \\
& \text { Transcription Regulator } \\
& \text { Transmembrane Receptor } \\
& \text { Other } \\
& \text { - Direct Relationship } \\
& \text {---Indirect Relationship } \\
& \text { colors: }
\end{aligned}
$$

Figure 7. Interaction of potential genes related to wool follicles development. Color indicates the expression of the gene in DR135 vs. D135. Red represents up-regulation of gene expression and green represents down-regulation of gene expression ( $\mid \log 2$ (fold change) $\mid>0.5)$. The intensity of green and red molecule colors indicates the degree of down or up-regulation, respectively. White molecules are not differential expression $(\mid \log 2$ (fold change) $\mid<0.5)$.

Secondary wool follicle development and branching are complex processes in Merino sheep. They involve many signaling molecules and regulatory factors that cooperate with each other. The Wnt and TGF- $\beta$ /BMP signaling pathways seem to be very important for the branching of SF, with both activation and inhibition coexisting to regulate the development of wool follicles. SFRP4, PITX1, BAMBI, and KRT16 warrant further investigation for their roles in the development of SF in Merino sheep.

\section{Conclusions}

Sub-M nutrition (75\% maintenance nutrition) has no significant effect on either ewe production and health or fetal development. There was no difference in the morphogenesis and development of fetal PF between the M and sub-M groups. However, the development and branching of SF were affected, such that SF density and S/P ratio were reduced following sub-maintenance feeding. Examination of the prenatal and postnatal development of wool follicles under pregnancy maintenance feeding and sub-maintenance nutrition in Merino sheep demonstrated that Wnt and TGF- $\beta$ /BMP signaling pathways play a crucial role in wool follicle development during days 105 to 135 of gestation. Further, SFRP4, PITX1, BAMBI, and KRT16 may be involved in SF branching and development in Merino sheep. 
Supplementary Materials: The following are available online at http://www.mdpi.com/2076-2615/10/6/1058/s1, Table S1: Energy standard and daily feeding intake of experimental ewe in maintenance group. Table S2: Changes of blood biochemical indexes in the $\mathrm{M}$ and sub-M group ewes. Table S3: Primer information of qRT-PCR. Table S4: Comparison of fetal body size of the $\mathrm{M}$ and Sub-M fetuses at different stages of gestation. Table S5: List of DEGs in D135 vs. D105. Table S6: List of DEGs in DR135 vs. D105. Table S7: List of DEGs in DR135 vs. D135. Figure S1: qRT-PCR validation of the 8 DEGs.

Author Contributions: Conceptualization: W.L.; Data curation: X.L. and S.H.; Formal analysis: X.L., L.C., C.L. and Z.L.; Funding acquisition: W.L.; Investigation: X.L., L.C., S.H., B.H. and M.Y.; Methodology: X.L., L.C., S.H., C.L., H.B., P.K., S.M., W.L. and M.L.; Project administration: M.L.; Resources: C.L., B.H., M.Y. and M.L.; Supervision: M.L.; Validation: Z.L.; Writing-original draft: X.L. L.C. and W.L.; Writing—review and editing: H.B., P.K. and S.M. All authors have read and agreed to the published version of the manuscript.

Funding: This study was funded by grant U1303284 from China National Natural Science Foundation a subcontract of grant 2013AA102506 from China National High Technology Research Development Program (863 Program). It was also in part supported by the Tianshan Xuesong Program of Xinjiang province (2018XS16).

Acknowledgments: We appreciate the experimental animal provided by the breeding farm in Urumqi of Xinjiang. We are also very grateful to the staff of the Key Laboratory of Animal Biotechnology, Xinjiang Academy of Animal Science.

Conflicts of Interest: The authors declare no conflict of interest. The funders had no role in the design of the study; in the collection, analyses, or interpretation of data; in the writing of the manuscript, or in the decision to publish the results.

\section{References}

1. Hardy, M.H.; Lyne, A.G. The histological development of the skin and wool in the Merino foetus. In Proceedings of the International Wool Textile Research Conference, Sydney, Australia, 1955; Commonwealth Scientific and Industrial Research Organization: Melbourne, Australia, 1955; pp. 26-31.

2. Bai, Y.; Tian, K.; Tian, Y.; Xu, X.; Huang, X. Correlation analysis for wool fiber diameter and identification traits in Chinese Merino sheep (Xinjiang type). Mod. J. Anim. Husb. Vet. Med. 2015, 9, 6-12.

3. Cordero, M.O.; Herrera, C.A.M.; García, J.M.V.; Stewart, C.A.; Nieto, C.A.R.; Alfaro, A.E.O.; Purvis, I.W.; Reyes, V.C.; Rangel, H.A.L.; Martin, G.B. Pregnancy and litter size, but not lamb sex, affect feed intake and wool production by Merino-type ewes. Animals 2019, 9, 214. [CrossRef]

4. Rasmussen, P.V.; Børsting, C.F. Effects of variations in dietary protein levels on hair growth and pelt quality in mink (Mustela vison). Can. J. Anim. Sci. 2000, 80, 633-642. [CrossRef]

5. Corbett, J.F. Hair conditioning. Cutis 1979, 23, 405-413.

6. Gebreselassie, G.; Berihulay, H.; Jiang, L.; Ma, Y.H. Review on genomic regions and candidate genes associated with economically important production and reproduction traits in sheep (Ovies aries). Animals 2019, 10, 33. [CrossRef]

7. Rishikaysh, P.; Dev, K.; Diaz, D.; Qureshi, W.M.; Filip, S.; Mokry, J. Signaling involved in hair follicle morphogenesis and development. Int. J. Mol. Sci. 2014, 15, 1647-1670. [CrossRef]

8. Gao, Y.; Wang, X.; Yan, H.; Zeng, J.; Ma, S.; Niu, Y.; Jiang, Y.; Chen, Y. Comparative transcriptome analysis of fetal skin reveals key genes related to hair follicle morphogenesis in cashmere goats. PLoS ONE 2016, 11, e0151118. [CrossRef]

9. Liu, Y.; Wang, L.; Li, X.; Han, W.; Yang, K.; Wang, H.; Zhang, Y.; Su, R.; Liu, Z.; Wang, R.; et al. High-throughput sequencing of hair follicle development-related micrornas in cashmere goat at various fetal periods. Saudi J. Biol. Sci. 2018, 25, 1494-1508. [CrossRef]

10. Zhu, Y.B.; Wang, Z.Y.; Yin, R.H.; Jiao, Q.; Zhao, S.J.; Cong, Y.Y.; Xue, H.L.; Guo, D.; Wang, S.Q.; Zhu, Y.X.; et al. A lncRNA-H19 transcript from secondary hair follicle of Liaoning cashmere goat: Identification, regulatory network and expression regulated potentially by its promoter methylation. Gene 2018, 641, 78-85. [CrossRef]

11. Bai, W.L.; Dang, Y.L.; Yin, R.H.; Jiang, W.Q.; Wang, Z.Y.; Zhu, Y.B.; Wang, S.Q.; Zhao, Y.Y.; Deng, L.; Luo, G.B.; et al. Differential expression of microRNAs and their regulatory networks in skin tissue of Liaoning cashmere goat during hair follicle cycles. Anim. Biotechnol. 2016, 27, 104-112. [CrossRef]

12. Geng, R.; Wang, L.; Wang, X.; Chen, Y. Cyclic expression of Lhx2 is involved in secondary hair follicle development in cashmere goat. Gene Expr. Patterns 2014, 16, 31-35. [CrossRef] [PubMed]

13. Schinckel, P.G. The post-natal development of the skin follicle population of a strain of Merino sheep. Aust. J. Agric. Res. 1955, 6, 215-224. [CrossRef] 
14. Fraser, A.S. Development of the skin follicle population in the Merino sheep. Aust. J. Agric. Res. 1954, 5, 737-744. [CrossRef]

15. Edwards, J.H.; Birtles, M.J.; Harris, P.M.; Parry, A.L.; Paterson, E.; Wickham, G.A.; McCutcheon, S.N. Preand post-natal wool follicle development and density in sheep of five genotypes. J. Agric. Sci. 1996, 126, 363-370. [CrossRef]

16. Wu, Y.; Yue, Y.; Guo, T.; Wang, T.; Guo, J.; Gui-ying, L.; Han, J.; Yang, M.; Liu, J.; Sun, X.; et al. Study on fetal skin hair follicle development and morphology of China super-fine Merino (Gansu type). Sci. Agric. Sin. 2013, 46, 1923-1931.

17. Schinckel, P.G.; Short, B.F. The influence of nutritional level during pre-natal and early post-natal life on adult fleece and body characters. Aust. J. Agric. Res. 1961, 12, 176-202. [CrossRef]

18. Shotr, B.F. Development of the secondary follicle population in sheep. Aust. J. Agric. Res. 1955, 6, 62-67.

19. Kelly, R.W.; Greeff, J.C.; Macleod, I. Lifetime changes in wool production of Merino sheep following differential feeding in fetal and early life. Aust. J. Agric. Res. 2006, 57, 867-876. [CrossRef]

20. Menzies, M.; Stockwell, S.; Brownlee, A.; Cam, G.; Ingham, A. Gene expression profiles of BMP4, FGF10 and cognate inhibitors, in the skin of foetal Merino sheep, at the time of secondary follicle branching. Exp. Dermatol. 2010, 18, 877-879. [CrossRef]

21. Zhou, Y.; Kipps, T.J.; Zhang, S. Wnt5a signaling in normal and cancer stem cells. Stem Cells Int. 2017, 2017, 5295286.

22. Tsai, S.Y.; Sennett, R.; Rezza, A.; Clavel, C.; Grisanti, L.; Zemla, R.; Najam, S.; Rendl, M. Wnt/ $\beta$-catenin signaling in dermal condensates is required for hair follicle formation. Dev. Biol. 2014, 385, 179-188. [CrossRef]

23. Kulessa, H.; Turk, G.; Hogan, B.L. Inhibition of Bmp signaling affects growth and differentiation in the anagen hair follicle. EMBO J. 2014, 19, 6664-6674. [CrossRef]

24. Oshimori, N.; Fuchs, E. Paracrine TGF- $\beta$ signaling counterbalances BMP-mediated repression in hair follicle stem cell activation. Cell Stem Cell 2012, 10, 63-75. [CrossRef]

25. Reddy, S.; Andl, T.; Bagasra, A.; Min, M.L.; Epstein, D.J.; Morrisey, E.E.; Millar, S.E. Characterization of Wnt gene expression in developing and postnatal hair follicles and identification of Wnt5a as a target of Sonic hedgehog in hair follicle morphogenesis. Mech. Dev. 2001, 107, 69-82. [CrossRef]

26. Guo, H.; Xing, Y.; Deng, F.; Yang, K.; Li, Y. Secreted Frizzled-related protein 4 inhibits the regeneration of hair follicles. PeerJ 2019, 6, e6153. [CrossRef]

27. Chen, C.C.; Murray, P.J.; Jiang, T.X.; Plikus, M.V.; Chang, Y.T.; Lee, O.K.; Widelitz, R.B.; Chuong, C.M. Regenerative hair waves in aging mice and extra-follicular modulators follistatin, Dkk1, and Sfrp4. J. Investig. Dermatol. 2014, 134, 2086-2096. [CrossRef]

28. Chen, C.C.; Chuong, C.M. Multi-layered environmental regulation on the homeostasis of stem cells: The saga of hair growth and alopecia. J. Dermatol. Sci. 2012, 66, 3-11. [CrossRef]

29. Bai, J.; Liu, Z.; Xu, Z.; Ke, F.; Zhang, L.; Zhu, H.; Lou, F.; Wang, H.; Fei, Y.; Shi, Y.L. Epigenetic downregulation of SFRP4 contributes to epidermal hyperplasia in psoriasis. J. Immunol. 2015, 194, 4185-4198. [CrossRef]

30. Maganga, R.; Giles, N.; Adcroft, K.; Unni, A.; Keeney, D.; Wood, F.; Fear, M.; Dharmarajan, A. Secreted Frizzled related protein-4 (sFRP4) promotes epidermal differentiation and apoptosis. Biochem. Biophys. Res. Commun. 2008, 377, 606-611. [CrossRef]

31. Uren, A.; Reichsman, F.; Anest, V.; Taylor, W.G.; Muraiso, K.; Bottaro, D.P.; Cumberledge, S.; Rubin, J.S. Secreted frizzled-related protein-1 binds directly to Wingless and is a biphasic modulator of Wnt signaling. J. Biol. Chem. 2000, 275, 4374-4382. [CrossRef]

32. Wang, J.S.; Infante, C.R.; Park, S.; Menke, D.B. PITX1 promotes chondrogenesis and myogenesis in mouse hindlimbs through conserved regulatory targets. Dev. Biol. 2017, 434, 186-195. [CrossRef]

33. Song, X.; Zhao, C.; Jiang, L.; Lin, S.; Bi, J.; Wei, Q.; Yu, L.; Zhao, L.; Wei, M. High PITX1 expression in lung adenocarcinoma patients is associated with DNA methylation and poor prognosis. Pathol. Res. Pract. 2018, 214, 2046-2053. [CrossRef]

34. Barut, F.; Udul, P.; Kokturk, F.; Kandemir, N.O.; Keser, S.H.; Ozdamar, S.O. Clinicopathological features and pituitary homeobox 1 gene expression in the progression and prognosis of cutaneous malignant melanoma. Kaohsiung J. Med. Sci. 2016, 32, 494-500. [CrossRef] 
35. Vidal, V.P.; Chaboissier, M.C.; Lützkendorf, S.; Cotsarelis, G.; Mill, P.; Hui, C.C.; Ortonne, N.; Ortonne, J.P.; Schedl, A. Sox9 is essential for outer root sheath differentiation and the formation of the hair stem cell compartment. Curr. Biol. 2005, 15, 1340-1351. [CrossRef] [PubMed]

36. Yamamizu, K.; Schlessinger, D.; Ko, M.S. SOX9 accelerates ESC differentiation to three germ layer lineages by repressing SOX2 expression through P21 (WAF1/CIP1). Development 2014, 141, 4254-4266. [CrossRef] [PubMed]

37. Song, L.; Cui, Y.; Yu, S.; Liu, P.; He, J. TGF- $\beta$ and HSP70 profiles during transformation of yak hair follicles from the anagen to catagen stage. J. Cell. Physiol. 2019, 234, 15638-15646. [CrossRef]

38. Tsuji, Y.; Denda, S.; Soma, T.; Raftery, L.; Momoi, T.; Hibino, T. A potential suppressor of TGF- $\beta$ delays catagen progression in hair follicles. J. Investig. Dermatol. Symp. Proc. 2003, 8, 65-68. [CrossRef]

39. Hibino, T.; Nishiyama, T. Role of TGF-beta2 in the human hair cycle. J. Dermatol. Sci. 2004, 35, 9-18. [CrossRef]

40. Foitzik, K.; Paus, R.; Doetschman, T.; Dotto, G.P. The TGF-beta2 isoform is both a required and sufficient inducer of murine hair follicle morphogenesis. Dev. Biol. 1999, 212, 278-289. [CrossRef]

41. Knight, C.; Simmons, D.; Gu, T.T.; Gluhak-Heinrich, J.; Pavlin, D.; Zeichner-David, M.; MacDougall, M. Cloning, characterization, and tissue expression pattern of mouse Nma/BAMBI during odontogenesis. J. Dent. Res. 2001, 80, 1895-1902. [CrossRef] [PubMed]

42. Sulayman, A.; Tursun, M.; Sulaiman, Y.; Huang, X.; Tian, K.; Tian, Y.; Xu, X.; Fu, X.; Mamat, A.; Tulafu, H. Association analysis of polymorphisms in six keratin genes with wool traits in sheep. Asian Australas. J. Anim. Sci. 2018, 31, 775-783. [CrossRef]

43. Wang, J.Q.; Hao, Z.Y.; Zhou, H.; Luo, Y.; Hu, J.; Liu, X.; Li, S.B.; Jonathan, H. A keratin-associated protein (KAP) gene that is associated with variation in cashmere goat fleece weight. Small Rumin. Res. 2018, 167, 104-109. [CrossRef]

44. Li, S.B.; Zhou, H.T.; Gong, H.; Zhao, F.F.; Wang, J.Q.; Liu, X.; Hu, J.; Luo, Y.Z.; Hickford, J.G.H. Identification of the ovine keratin-associated protein 21-1 gene and its association with variation in wool traits. Animals 2019, 9, 450. [CrossRef] [PubMed]

45. Bai, L.R.; Wang, J.; Zhou, H.T.; Gong, H.; Tao, J.Z.; Hickford, J.G.H. Identification of ovine KRTAP28-1 and its association with wool fibre diameter. Animals 2019, 9, 142. [CrossRef]

46. Rogers, M.A.; Winter, H.; Langbein, L.; Wolf, C.; Schweizer, J. Characterization of a 300 kbp region of human DNA containing the type II hair keratin gene domain. J. Investig. Dermatol. 2000, 114, 464-472. [CrossRef]

47. Tai, W.T.; Chen, Y.L.; Chu, P.Y.; Chen, L.J.; Hung, M.H.; Shiau, C.W.; Huang, J.W.; Tsai, M.H.; Chen, K.F. Protein tyrosine phosphatase 1B dephosphorylates PITX1 and regulates p120RasGAP in hepatocellular carcinoma. Hepatology 2016, 63, 1528-1543. [CrossRef] [PubMed] 